\title{
Lupus Vulgaris Involving Ear Lobule
}

\section{Poonam Adlakha ${ }^{1 *}$ and Mohit Nagpal ${ }^{2}$}

${ }^{1}$ Department of Histopathology, Postgraduate Institute of Medical Education and Research, Chandigarh, India ${ }^{2}$ Department of Dermatology, Venereology, and Leprology Postgraduate Institute of Medical Education and Research, Chandigarh, India

\section{Dear Sir}

Lupus vulgaris is a chronic, progressive, post primary, paucibacillary form of cutaneous tuberculosis occurring in immunocompetent individuals and is the most common form of tuberculosis in adults in India. ${ }^{[1,2]}$ Face is affected less often and the buttocks and trunk are involved more frequently. ${ }^{[3]}$ A 35 year old female presented to the dermatology out-patient with an ulcerated lesion on right side ear lobule. Initially, the lesion was nodular, increasing in size despite treatment with intermittent courses of antibiotics (Amoxycillin-clavulinic acid)

Examination revealed an ulcerated plaque over the lobule of right ear, having erythematous base and showing hemorrhagic crusts at places (Figure 1) Surrounding skin was erythematous and oedematous. The lesion was non- tender and no satellite lesions noted. There was no significant lymphadenopathy. Peripheral nerve trunks were not palpable. There were no other associated symptoms, history of trauma, vaccination or medical history of tuberculosis. All the routine haematological and biochemical investigations were normal. Chest radiograph did not reveal any significant pathology. Skin biopsy from the edge of the lesion was carried out. Skin biopsy on $\mathrm{H}$ \& E stain revealed hyperkeratotic epidermis with multiple non- caseating granulomas comprising of epithelioid cells,

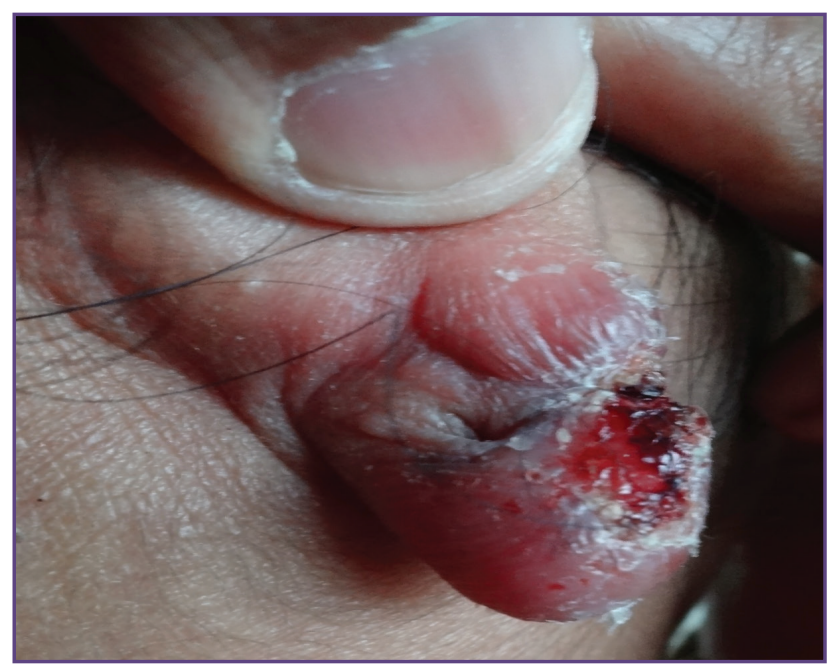

Fig. 1: Ulcerated plaque over the lobule of right ear. lymphocytes, histiocytes and Langhans giant cells in the dermis (Figure 2, 3)

The histomorphological features were suggestive of Lupus vulgaris. Patient was started on 4 drug Anti-tubercular therapy under Category I Rifampicin, Pyrazinamide, Isoniazid and Ethambutol)

Lupus vulgaris originates from an underlying focus of tuberculosis, bone, joint or lymph node and arises by either contiguous extension from underlying tissue or by lymphatic or hematogenous spread. In cases of clinically inapparent underlying focus, reactivation of some latent cutaneous focus secondary to previous silent bacteremia is considered. ${ }^{[4]}$

The conventional morphological forms of Lupus vulgaris are broadly categorised into plaque, ulcerative and mutilating, vegetating, tumor-like and popular-nodular forms. ${ }^{[1]}$ However, atypical forms at unconventional sites are becoming increasingly common and pose great diagnostic dilemmas.

Two cases of Lupus vulgaris involving face; cheek and periorbital area have been described by Khandpur S et al [5] presenting as periorbital cellulitis and basal cell carcinoma respectively. PCR performed on tissue sections were positive in both of the cases. Chakravarti A et al[ ${ }^{[6]}$ reported

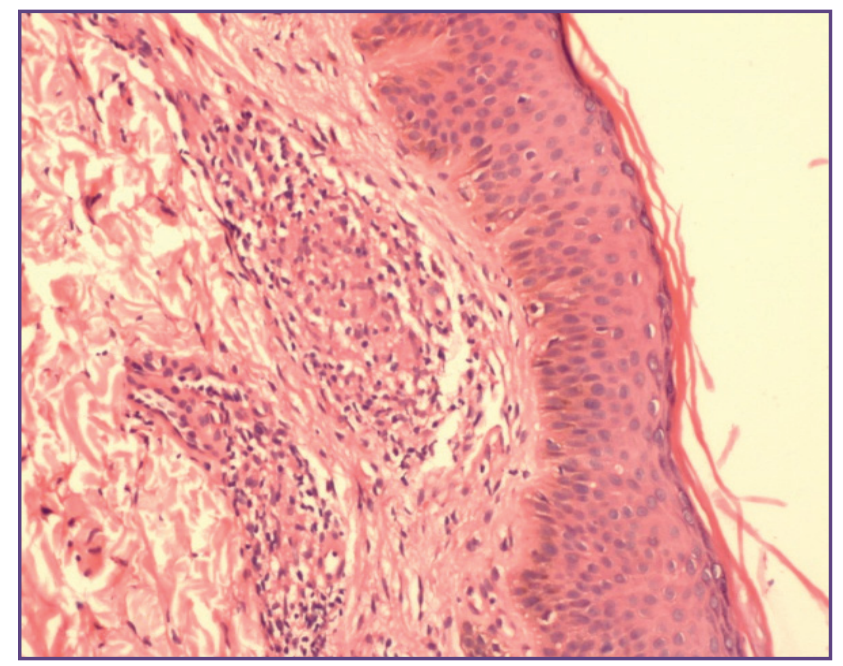

Fig. 2: Microphotograph showing non-caseating granulomas in the dermis (H\&E, 100X) 


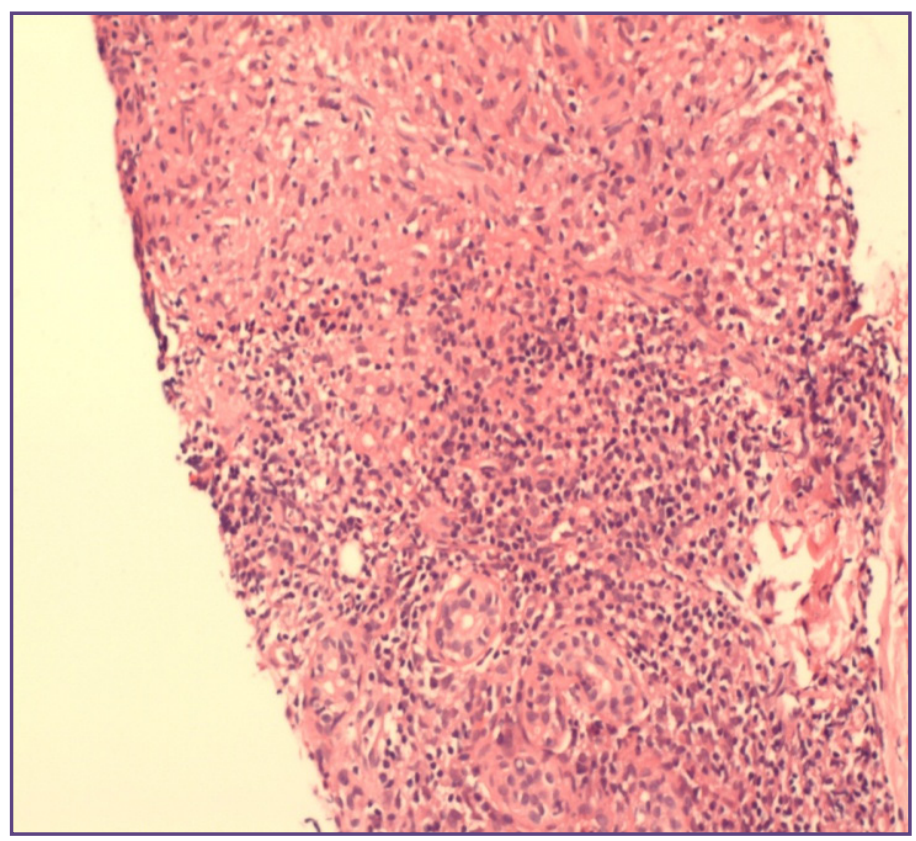

Fig. 3 : Tuberculoid granuloma comprised of epithelioid cells, lympho-histiocytes and ill-defined Langhans giant cells (H\&E, 400X).

a case involving the external nose in an immunocompetent elderly male patient with a 9 month history of nodule ulcerative lesion extending upto the naso-facial groove. Apart from raised ESR, TB IgG antibodies were positive.

There is only isolated report involving the ear in case of a nine year old girl, who presented as erythematous plaques on both ears and surrounding area following ear piercing. ${ }^{[7]}$

Cutaneous tuberculosis continues to be present over atypical sites and in various morphological forms; making diagnoses difficult. Histopathological examination and other modalities including PCR and ELISA should be used for confirmation.

\section{Reference}

1. Yates VM. Mycobacterial infections. In: Burns T, Breathnach S, Cox N, Griffiths C (Eds). Textbook of Dermatology, 8th ed. Oxford: Blackwell, 2010; V.2, 31.16.
2. Kumar B, Muralidhar S. Cutaneous tuberculosis: a twentyyear prospective series. Int J Tuberc Lung Dis 1999; 3: 494-500.

3. Sehgal VN, Waugh SA. Cutaneous tuberculosis. Current concepts. Int J Dermatol 1990; 29:237-52.

4. Marcoval J, Servitje O, Moreno A et al. Lupus vulgaris: clinical, histopathologic and bacteriologic study of 10 cases. J Am Acad Dermatol 1992; 26: 404-7.

5. Khandpur S and Reddy B. Lupus vulgaris: unusual presentations over the face. Journal of the European Academy of Dermatology and Venereology 2003; 17: 706-10.

6. Chakravarti A, Dhawan R, Shashidhar T.B, Sahni J.K. Lupus vulgaris of external nose. Indian J Tuberc 2006; 53:220-2.

7. Kumar P, Mondal A, Lal NR, Gharami RC. Lupus vulgaris in a child: A complication of ear piercing. Indian J Dermatol Venereol Leprol 2014; 80:97.

*Corresponding author:

Dr Poonam Adlakha, 648/30 Adarsh Nagar, Rohtak, India

Phone: +91 8813845435

Email: poonam945@yahoo.com

Financial or other Competing Interests: None.

Date of Submission : 24.09.2016

Date of Acceptance : 14.12.2016

Date of Publication : 19.02.2017 